was $4 \%(n=6)$. Details of these cases can be seen in table 1 . Isolated IUGR, isolated prematurity, uncomplicated multiple birth and a gestational age of greater than 34 weeks were not associated with a severe abnormality.

Conclusions Our study found a $4 \%$ severe anomaly rate in moderately preterm infants screened with CUS. But we found the infants in our study with a severe anomaly had clinically significant indications for scanning other than their prematurity and we believe they would have required scanning no matter their gestational age. These results suggest targeted screening may have been adequate to identify those at high risk. Further studies are required to delineate the true overall rate of CUS abnormalities in late preterm infants and to correlate these with neurodevelopmental outcomes.

\section{P0-0616 A MULTICENTER, RANDOMISED, CONTROLLED TRIAL OF OSTEOPATHIC MANIPULATIVE TREATMENT ON PRETERM INFANTS}

${ }^{1} \mathrm{~F}$ Cerritelli, ${ }^{1} \mathrm{G}$ Pizzolorusso, ${ }^{1} \mathrm{C}$ Renzetti, ${ }^{1} \mathrm{~V}$ Cozzolino, ${ }^{1} \mathrm{M}$ D'Orazio, ${ }^{1} \mathrm{MC}$ Lupacchini, ${ }^{1} \mathrm{~B}$ Marinelli, ${ }^{1} \mathrm{~A}$ Accorsi, ${ }^{1} \mathrm{C}$ Lucci, ${ }^{1} \mathrm{~J}$ Lancellotti, ${ }^{2} \mathrm{~S}$ Ballabio, ${ }^{2} \mathrm{C}$ Castelli, ${ }^{2} \mathrm{D}$ Molteni, ${ }^{2} \mathrm{R}$ Besana, ${ }^{3} \mathrm{~L}$ Tubaldi, ${ }^{3} \mathrm{FP}$ Perri, ${ }^{4} \mathrm{P}$ Fusilli, ${ }^{4} \mathrm{C} \mathrm{D}^{\prime}$ Incecco, ${ }^{1} \mathrm{G}$ Barlafante. ${ }^{1}$ Research, Accademia Italiana Osteopatia Tradizionale, Pescara, Italy; ${ }^{2}$ Neonatology, Desio Hospital, Desio, Italy; ${ }^{3}$ Neonatology, Macerata Hospital, Macerata, Italy; ${ }^{4}$ Neonatology, Pescara Hospital, Pescara, Italy

\subsection{6/archdischild-2014-307384.1258}

Background It is still uncertain if osteopathic manipulative treatment improves preterm clinical outcomes.

Methods The present multi-centre randomised single blind parallel group clinical trial enrolled newborns who met the criteria for gestational age between 29 and 37 weeks without any congenital complication from 3 different public neonatal intensive care units. Preterm infants were randomly assigned to usual prenatal care (control group) or osteopathic manipulative treatment (study group). The primary outcome was the mean difference in length of stay between groups.

Results A total of 695 newborns were randomly assigned to the study group $(\mathrm{n}=352)$ and to the control group $(\mathrm{n}=343)$. A statistical significant difference was observed between the two groups for the primary outcome (13.8 and 17.5 days for the study and control group respectively, $\mathrm{p}<0.001$, effect size: $0.31)$. Multivariate analysis showed a reduction of the length of stay of 3.9 days $(-5.5$ to $-2.3, \mathrm{p}<0.001)$. Furthermore, there were significant reductions with treatment as compared to usual care in cost (difference between study and control group: $1,586.01 € ; 1,087.18$ to $6,277.28 ; \mathrm{p}<0.001$ ) but not in daily weight gain. The relative risk of developing any respiratory problem during the study period was 0.53 (0.42 to 0.64$)$. Moreover, the estimated research period attributable risk was $47 \%$. There were no complications associated to the intervention.

Conclusions Osteopathic treatment reduced significantly the number of days of hospitalisation and costs on a large cohort of preterm infants.

\section{P0-0617 QUALITY IMPROVEMENT REVIEW OF UNINTERRUPTED POWER SUPPLY FOR CRITICAL CARE EQUIPMENT IN NEONATAL UNITS IN NORTHERN IRELAND}

${ }^{1} \mathrm{~K}$ Courtenay, ${ }^{1} \mathrm{AM}$ McClean, ${ }^{1} \mathrm{C}$ McFeely, ${ }^{1} \mathrm{M}$ Anandarajan, ${ }^{2} \mathrm{R}$ Tubman. ${ }^{1}$ Paediatrics, Ulster Hospital Dundonald, Belfast, UK; ${ }^{2}$ Neonatology, Royal Maternity Hospital, Belfast, UK

10.1136/archdischild-2014-307384.1259
Introduction/background Intensive care equipment is dependent on an uninterrupted power supply (UPS) in the event of power failure. It is important that medical and nursing staff have a basic knowledge of critical care equipment and are aware of guidelines/protocols in place, to prevent possible harm to patients in the event of mains power failure.

Method A questionnaire audit was performed amongst medical and nursing staff in all five neonatal intensive care units in Northern Ireland. The aim was to look at current practice, identify areas of staff knowledge regarding UPS principles and how to maintain critical care equipment in the event of power failure. Results Eighty-eight (44\%) questionnaires were returned. The results were as follows:

- $73 \%$ of respondents were aware their unit had UPS.

- $25 \%$ were unsure which items should be plugged into UPS.

- $58 \%$ were aware of the presence of back up batteries in critical care equipments.

- In the event of power failure $81 \%$ of respondents said they would contact the nurse in charge whilst a technician was the next frequent point of contact, but there was no clear procedure beyond the initial contact.

Conclusions The results showed wide variation in staff knowledge regarding the availability of UPS, the presence of an internal back up battery and which equipment should be plugged into UPS circuits. There was also lack of guidance regarding whom to contact in the event of power failure. This study resulted in training, staff education and development of guidance regarding UPS circuits.

\section{PO-0618 FIRST PERSON PERSPECTIVE ENHANCES PROCEDURAL METRICS}

K Cunningham, G Hawkes, L O'Connell, E Dempsey. Neonatal Unit, Cork University Maternity Hospital, Cork, Ireland

\subsection{6/archdischild-2014-307384.1260}

Background Procedural videos are used to enhance training. Recording of procedures and video coaching may enhance procedural skills. These typically are from third person perspective.

Aim To determine if first person perspective procedural video recording of pigtail catheter insertion in a rabbit model enhances procedural based metric development compared to standard video recording.

Methods Four neonatologists were filmed in real time using the trocar technique to insert a pigtail catheter chest drain in a rabbit model, under sterile conditions. Each participant was recorded using a stationary recording device, while also wearing point of site video recording glasses (similar to spectacles without lenses). Stationary video recordings were considered to provide third person perspective. Video recording glasses provided first person perspective. All videos were independently scored on a predefined 15 item procedural metric.

Results Third person video recording failed to identify $40 \%$ $(20-50 \%)$ of key intra-procedural components; inserting the needle, using the trocar, inserting the guide wire and dilator. Where as, only $10 \%(0-10 \%)$ was not identified from first person perspective.

Video recording, both first and third person, highlighted other components not previously identified in the original metric, such as important interactions with the assistant. Consistent opinions contributed towards compiling a new best-practice 